\title{
A systematic review identifying common data items in neonatal trials and assessing their completeness in routinely recorded United Kingdom national neonatal data
}

\author{
Sena Jawad ${ }^{1}$, Neena Modi ${ }^{1}$, A. Toby Prevost ${ }^{2}$ and Chris Gale ${ }^{1 *}$ (D)
}

\begin{abstract}
Background: We aimed to test whether a common set of key data items reported across high-impact neonatal clinical trials could be identified, and to quantify their completeness in routinely recorded United Kingdom neonatal data held in the National Neonatal Research Database (NNRD).

Methods: We systematically reviewed neonatal clinical trials published in four high-impact medical journals over 10 years (2006-2015) and extracted baseline characteristics, stratification items and potential confounders used to adjust primary outcomes. Completeness was examined using data held in the NNRD for identified data items, for infants admitted to neonatal units in 2015. The NNRD is a repository of routinely recorded data extracted from neonatal Electronic Patient Records (EPR) of all admissions to National Health Service (NHS) Neonatal Units in England, Wales and Scotland. We defined missing data as an empty field or an implausible value. We reported common data items as frequencies and percentages alongside percentages of completeness.
\end{abstract}

Results: We identified 44 studies involving 32,095 infants and 126 data items. Fourteen data items were reported by more than $20 \%$ of studies. Gestational age (95\%), sex (93\%) and birth weight (91\%) were the most common baseline data items. The completeness of data in the NNRD was high for these data with greater than 90\% completeness found for 9 of the 14 most common items.

Conclusion: High-impact neonatal clinical trials share common data items. In the United Kingdom, these items can be obtained at a high level of completeness from routinely recorded data held in the NNRD. The feasibility and efficiency using routinely recorded EPR data, such as that held in the NNRD, for clinical trials, rather than collecting these items anew, should be examined.

Trial registration: PROSPERO registration number CRD42016046138. Registered prospectively on 17 August 2016.

Keywords: Common data items, Data quality, NNRD, Efficient trials, Electronic patient records, Electronic health records, Neonatal clinical trials

\footnotetext{
* Correspondence: christopher.gale@imperial.ac.uk

${ }^{1}$ Neonatal Medicine, School of Public Health, Faculty of Medicine, Imperial College London, Chelsea and Westminster Hospital Campus, London SW10 $9 \mathrm{NH}, \mathrm{UK}$

Full list of author information is available at the end of the article
}

(c) The Author(s). 2019 Open Access This article is distributed under the terms of the Creative Commons Attribution 4.0 International License (http://creativecommons.org/licenses/by/4.0/), which permits unrestricted use, distribution, and reproduction in any medium, provided you give appropriate credit to the original author(s) and the source, provide a link to the Creative Commons license, and indicate if changes were made. The Creative Commons Public Domain Dedication waiver (http://creativecommons.org/publicdomain/zero/1.0/) applies to the data made available in this article, unless otherwise stated. 


\section{Introduction}

High-quality randomised controlled trials are considered the gold standard research approach to identify causality or demonstrate treatment efficacy. There are many treatment uncertainties in neonatal practice [1] that would benefit from being subjected to high-quality randomised clinical trials [2]. However, the high cost of undertaking large and methodologically robust trials [3] means that only a small number are undertaken each year: the median cost of randomised controlled trials was estimated between US $\$ 43$ and US $\$ 103,254$ per participant [4] and publicly funded pragmatic neonatal trials cost $£ 1.5-2$ million [5]. A key driver of cost in clinical trials is data collection; the mean costs of trial data collection using conventional Case Record Forms have been estimated to be $€ 1135$ per participant [6]. More efficient collection; for example, using electronic Case Record Forms [6] and routinely available clinical data [7], provide opportunities to reduce costs and facilitate neonatal trials to improve the limited evidence base upon which much of neonatal care currently relies.

Methods to increase the efficiency of clinical trial data collection have been described by organisations such as the Institute of Medicine [8] and the Clinical Trials Transformation Initiative [9]; these include a targeted collection of common core data items, and extraction of trial data from existing sources, such as Electronic Patient Record (EPR) systems or disease registries; these approaches are most likely to be applicable to pragmatic trials [10]. The use of existing 'real-world' data sources such as these provides additional advantages: they can provide up-to-date incidence estimates for baseline and outcome event rates to better inform sample size calculations, and the accuracy and completeness of key data items can be estimated in advance from historical data to inform trial feasibility at the planning stage, and address widely held concerns about poor quality of data from existing sources [11]. However, because not all data items held within a routinely recorded database or registry will be relevant to clinical trials, the data items that are 'core' [9] for clinical trials in a particular clinical area need to be established. Established approaches exist for the definition of Core Outcome Sets [12], but none for core non-outcome data for clinical trials; for example, baseline or background data, and items used in randomisation.

An increasing proportion of neonatal Cochrane reviews are inconclusive because of insufficient highquality data from randomised trials [2]. Neonatal care in the United Kingdom is well placed to develop large, efficient trials that use existing data: all infants admitted for National Health Service (NHS) neonatal care in England, Scotland and Wales have clinical data recorded in a summary EPR system as part of routine clinical care, and predefined data [13] are extracted to form the
National Neonatal Research Database (NNRD). The effectiveness and efficiency of using routinely recorded clinical data, held in the NNRD for data-enabled neonatal trials, are currently being investigated [14]. We hypothesised that a set of common data items have been reported across neonatal trials that impact clinical practice; the aim of this study was to identify common neonatal data items. As there is no established approach for the identification of common baseline data items we undertook a systematic review to identify baseline data items reported in neonatal trials. A secondary aim was to quantify the completeness of these commonly reported items in the NNRD to inform whether this could be used as the sole or principal data source for clinical trials.

\section{Methods}

\section{Systematic review}

To identify data commonly reported in neonatal trials we conducted a systematic review of neonatal clinical trials published in high-impact journals. We developed a protocol with explicitly defined objectives, information to be extracted, and statistical methods. We prospectively registered the protocol with PROSPERO International Prospective Register of Systematic Reviews, registration number CRD42016046138 (https://www.crd. york.ac.uk/prospero), registered on 17 August 2016.

We searched the four most highly cited general medical journals that publish neonatal trials [15] (New England Journal of Medicine, Lancet, British Medical Journal and Journal of the American Medical Association) over a 10year period from 1 January 2006 to 31 December 2015, using the PubMed database. The PubMed search strategy is described in Additional file 1. We extracted randomised clinical trials written in English that tested an intervention delivered to newborn infants in a neonatal unit setting, with no restriction on the disease area or treatment type. Prior to data extraction we changed the inclusion criteria for studies to include trials of infants born at more than 34 gestational weeks, so that the results would be more generalisable to neonatal trials. We did not include trials where an intervention was applied to a pregnant mother and infant outcomes were reported. Two authors (SJ and CG) independently performed the screening of each potentially relevant record and reviewed full text where necessary to assess eligibility. Discrepancies between the authors were resolved through discussion.

Two authors (SJ, CG) independently extracted the following items from included clinical trials: baseline items, items used in stratification or minimisation (randomisation), and items used to adjust primary outcomes. Other study characteristics that we extracted included whether the trial was multicentre and whether it involved preterm or term infants. Outcome data were not extracted as these are the subject of other parallel work [16]. A 
comprehensive list of reported data items and frequencies was extracted. Items were combined where appropriate; for example, administration of different medications was combined into the item 'medications'. Preterm studies were defined as studies involving babies with a gestational age of less than 37 weeks or weighing less than $1500 \mathrm{~g}$ and term studies as studies on babies born at or above 37 weeks' gestation. A formal risk of bias assessment was not conducted as the interest of this study was limited to the data collected, not the interventions or the measure of efficacy.

\section{Data completeness}

Data completeness in the NNRD was examined for infants born in England, Scotland and Wales during the period 1 January 12015 to 31 December 2015 for the first seven postnatal days. The NNRD contains over 400 different data per each baby; data held in the NNRD are extracted from individual infants' EPR data routinely recorded by healthcare professionals as part of clinical care. Details of the Neonatal Dataset are searchable at the following webpage [13] and descriptive data for infants within the NNRD are available here [17]. We calculated the completeness in the NNRD of each data item reported by at least $20 \%$ of clinical trials included in the systematic review.

We defined incompleteness as an empty field or an implausible value. Where an item identified through the systematic review (for example, birth weight) directly matched a corresponding NNRD field, the completeness of these items was directly calculated. Where an item identified in the systematic review mapped to several fields in the NNRD (for example, respiratory support, identified in the systematic review, maps to several NNRD fields, including use of respiratory support, mode of ventilation, non-invasive respiratory support, nitric oxide, tracheostomy, surfactant [13], completeness was determined by at least one value that was not missing or implausible (according to the neonatal dataset data dictionary definition) over the multiple possible NNRD fields.

\section{Results}

\section{Systematic review}

We identified 161 articles in the literature search. We excluded 117 articles leaving 44 eligible to be included in the review (Fig. 1). Twenty-nine studies included only preterm babies, six only term babies and nine studies included both term and preterm babies (Table 1). The majority of studies (91\%) were multicentre trials and overall included 30,968 participants (Table 1).

The median number of baseline data items reported in the 44 included trials was 12 . Gestational age, sex and birth weight were collected as baseline items for 42 of 44 studies (Table 2). Fourteen data items were reported by at least $20 \%$ of studies; 66 baseline data items were reported by one study alone (Additional file 2: Table S1). No study reported all 14 of the most common data items.

Sixteen stratification items were reported by 35 trials. Neonatal unit identifier (57\%) and gestational age (39\%) were the most common items used for stratification

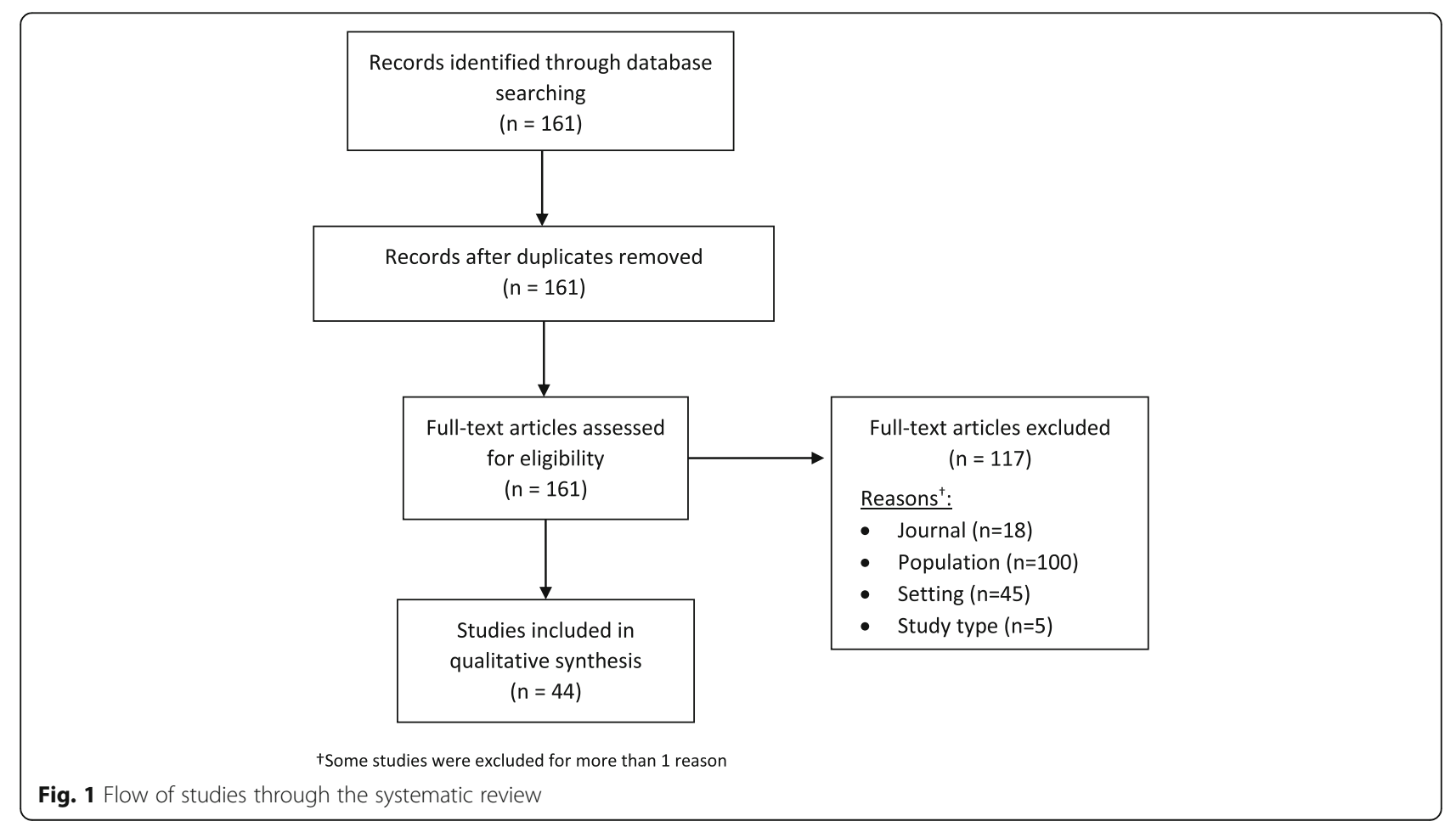


Table 1 The identified studies and their characteristics

\begin{tabular}{|c|c|c|c|c|c|c|c|c|}
\hline $\begin{array}{l}\text { Author and } \\
\text { year }\end{array}$ & Title & $N^{a}$ & Intervention arm & Comparator arm & $\begin{array}{l}\text { Single/ } \\
\text { multiple } \\
\text { centre } \\
\text { trial }\end{array}$ & $\begin{array}{l}\text { Age/ } \\
\text { weight } \\
\text { Inclusion } \\
\text { criteria of } \\
\text { participants }\end{array}$ & $\begin{array}{l}\text { Infant } \\
\text { age } \\
\text { group }\end{array}$ & Disease area \\
\hline $\begin{array}{l}\text { Azzopardi } \\
2009[18]\end{array}$ & $\begin{array}{l}\text { Moderate hypothermia to treat } \\
\text { perinatal asphyxial encephalopathy }\end{array}$ & 325 & $\begin{array}{l}\text { Total body } \\
\text { cooling and } \\
\text { intensive care }\end{array}$ & Intensive care & Multiple & $\begin{array}{l}\geq 36 \\
\text { weeks' } \\
\text { gestation }\end{array}$ & Term & Neurological \\
\hline $\begin{array}{l}\text { Azzopardi } \\
2014[19]\end{array}$ & $\begin{array}{l}\text { Effects of hypothermia for perinatal } \\
\text { asphyxia on childhood outcomes }\end{array}$ & 325 & $\begin{array}{l}\text { Standard care } \\
\text { with hypothermia }\end{array}$ & Standard care & Multiple & $\geq 36$ weeks & Term & Neurological \\
\hline $\begin{array}{l}\text { Ballard } 2006 \\
\text { [20] }\end{array}$ & $\begin{array}{l}\text { Inhaled nitric oxide in preterm infants } \\
\text { undergoing mechanical ventilation }\end{array}$ & 582 & Nitric oxide & Placebo & Multiple & $<32$ weeks & Preterm & Respiratory \\
\hline $\begin{array}{l}\text { Bassler } 2015 \\
{[21]}\end{array}$ & $\begin{array}{l}\text { Early inhaled budesonide for the } \\
\text { prevention of bronchopulmonary } \\
\text { dysplasia }\end{array}$ & 856 & $\begin{array}{l}\text { Early inhaled } \\
\text { budesonide }\end{array}$ & Placebo & Multiple & $\begin{array}{l}23^{+0} \text { to } \\
27^{+6} \\
\text { weeks }^{\text {+days }}\end{array}$ & Preterm & Respiratory \\
\hline $\begin{array}{l}\text { Baud } 2016 \\
{[22]}\end{array}$ & $\begin{array}{l}\text { Effect of early low-dose hydrocortisone } \\
\text { on survival without bronchopulmonary } \\
\text { dysplasia in extremely preterm infants } \\
\text { (PREMILOC): a double-blind, placebo- } \\
\text { controlled, multicentre randomised trial }\end{array}$ & 521 & Hydrocortisone & Placebo & Multiple & $\begin{array}{l}24^{+0} \text { to } \\
27^{+6} \\
\text { weeks }^{\text {+days }}\end{array}$ & Term & Respiratory \\
\hline $\begin{array}{l}\text { Beardsall } \\
2008[23]\end{array}$ & $\begin{array}{l}\text { Early insulin therapy in very-low- } \\
\text { birth-weight infants }\end{array}$ & 386 & Early insulin & $\begin{array}{l}\text { Standard } \\
\text { neonatal care }\end{array}$ & Multiple & $<1500 \mathrm{~g}$ & Preterm & $\begin{array}{l}\text { Other- } \\
\text { metabolic/ } \\
\text { endocrine }\end{array}$ \\
\hline $\begin{array}{l}\text { Benjamin } \\
2014[24]\end{array}$ & $\begin{array}{l}\text { Effect of fluconazole prophylaxis on } \\
\text { candidiasis and mortality in } \\
\text { premature infants, a randomized } \\
\text { clinical trial }\end{array}$ & 361 & Fluconazole & Placebo & Multiple & $<750 \mathrm{~g}$ & Preterm & Infection \\
\hline $\begin{array}{l}\text { Brocklehurst } \\
2011[25]\end{array}$ & $\begin{array}{l}\text { Treatment of neonatal sepsis with } \\
\text { intravenously administered immune } \\
\text { globulin }\end{array}$ & 3493 & $\begin{array}{l}\text { Polyvalent lgG } \\
\text { immune globulin }\end{array}$ & Placebo & Multiple & $<1500 \mathrm{~g}$ & Preterm & Infection \\
\hline $\begin{array}{l}\text { Carlo } 2010 \\
{[26]}\end{array}$ & $\begin{array}{l}\text { Target ranges of oxygen saturation in } \\
\text { extremely preterm infants }\end{array}$ & 1316 & $\begin{array}{l}\text { Oxygen saturation } \\
85-89 \%\end{array}$ & $\begin{array}{l}\text { Oxygen } \\
\text { saturation } 91- \\
95 \%\end{array}$ & Multiple & $\begin{array}{l}24^{+0} \text { to } \\
27^{+6} \\
\text { weeks }^{+ \text {days }}\end{array}$ & Preterm & Respiratory \\
\hline $\begin{array}{l}\text { Carr } 2009 \\
{[27]}\end{array}$ & $\begin{array}{l}\text { Granulocyte-macrophage colony } \\
\text { stimulating factor administered as } \\
\text { prophylaxis for reduction of sepsis in } \\
\text { extremely preterm, small-for- } \\
\text { gestational age neonates (PROGRAMS): } \\
\text { a single-blind, multicentre randomised } \\
\text { controlled trial }\end{array}$ & 280 & $\begin{array}{l}\text { Granulocyte- } \\
\text { macrophage } \\
\text { colony } \\
\text { stimulating factor }\end{array}$ & Standard care & Multiple & $\leq 31$ weeks & Preterm & Infection \\
\hline $\begin{array}{l}\text { Ceelie } 2013 \\
\text { [28] }\end{array}$ & $\begin{array}{l}\text { Effect of intravenously administered } \\
\text { paracetamol on postoperative } \\
\text { morphine requirements in neonates } \\
\text { and infants undergoing major } \\
\text { noncardiac surgery }\end{array}$ & 71 & $\begin{array}{l}\text { Continuous } \\
\text { morphine }\end{array}$ & $\begin{array}{l}\text { Intermittent } \\
\text { intravenously } \\
\text { administered } \\
\text { paracetamol }\end{array}$ & Single & $\begin{array}{l}>36^{+1} \\
\text { week }^{+ \text {days }} \\
\text { to } 1 \text { year }\end{array}$ & Term & Other- pain \\
\hline $\begin{array}{l}\text { Costeloe } \\
2016[29]\end{array}$ & $\begin{array}{l}\text { Bifidobacterium breve BBG-001 in very } \\
\text { preterm infants: a randomised controlled } \\
\text { phase } 3 \text { trial }\end{array}$ & 1310 & $\begin{array}{l}\text { Probiotic } B \text { breve } \\
\text { BBG-001 }\end{array}$ & Placebo & Multiple & $\begin{array}{l}23^{+0} \text { to } \\
30^{+6} \\
\text { weeks }^{\text {ddays }}\end{array}$ & Preterm & Infection \\
\hline $\begin{array}{l}\text { Davidson } \\
2016[30]\end{array}$ & $\begin{array}{l}\text { Neurodevelopmental outcome at } 2 \\
\text { years of age after general anaesthesia } \\
\text { and awake-regional anaesthesia in } \\
\text { infancy (GAS): an international multicentre, } \\
\text { randomised controlled trial }\end{array}$ & 719 & $\begin{array}{l}\text { Awake-regional } \\
\text { anaesthesia }\end{array}$ & $\begin{array}{l}\text { General } \\
\text { anaesthesia }\end{array}$ & Multiple & $\begin{array}{l}\geq 26 \text { weeks } \\
\text { to } 60 \\
\text { weeks }\end{array}$ & Both & $\begin{array}{l}\text { Other- } \\
\text { sedation/ } \\
\text { anaesthesia }\end{array}$ \\
\hline $\begin{array}{l}\text { Fergusson } \\
2012[31]\end{array}$ & $\begin{array}{l}\text { Effect of fresh red blood cell } \\
\text { transfusions on clinical outcomes in } \\
\text { premature, very-low-birth-weight } \\
\text { infants }\end{array}$ & 377 & $\begin{array}{l}\text { Fresh red blood } \\
\text { cell transfusions }\end{array}$ & $\begin{array}{l}\text { Standard red } \\
\text { blood cell } \\
\text { transfusions }\end{array}$ & Multiple & $<1250 \mathrm{~g}$ & Preterm & $\begin{array}{l}\text { Other- } \\
\text { haematological }\end{array}$ \\
\hline $\begin{array}{l}\text { Finer } 2010 \\
{[32]}\end{array}$ & $\begin{array}{l}\text { Early continuous positive airway } \\
\text { pressure (CPAP) versus surfactant in } \\
\text { extremely preterm infants }\end{array}$ & 1316 & $\begin{array}{l}\text { Intubation and } \\
\text { surfactant }\end{array}$ & $\begin{array}{l}\text { Continuous } \\
\text { positive airway } \\
\text { pressure }\end{array}$ & Multiple & $\begin{array}{l}24^{+0} \text { to } \\
27^{+6} \\
\text { weeks }^{\text {+days }}\end{array}$ & Preterm & Respiratory \\
\hline Fivez 2016 & Early versus late parenteral nutrition & 1440 & Late parenteral & Early parenteral & Multiple & Term & Term & Other- \\
\hline
\end{tabular}


Table 1 The identified studies and their characteristics (Continued)

\begin{tabular}{|c|c|c|c|c|c|c|c|c|}
\hline $\begin{array}{l}\text { Author and } \\
\text { year }\end{array}$ & Title & $N^{a}$ & Intervention arm & Comparator arm & $\begin{array}{l}\text { Single/ } \\
\text { multiple } \\
\text { centre } \\
\text { trial }\end{array}$ & $\begin{array}{l}\text { Age/ } \\
\text { weight } \\
\text { Inclusion } \\
\text { criteria of } \\
\text { participants }\end{array}$ & $\begin{array}{l}\text { Infant } \\
\text { age } \\
\text { group }\end{array}$ & Disease area \\
\hline [33] & in critically ill children & & nutrition & nutrition & & $\begin{array}{l}\text { newborns } \\
\text { to } 17 \text { years }\end{array}$ & & nutrition \\
\hline $\begin{array}{l}\text { Gopel } 2011 \\
\text { [34] }\end{array}$ & $\begin{array}{l}\text { Avoidance of mechanical ventilation } \\
\text { by surfactant treatment of } \\
\text { spontaneously breathing preterm } \\
\text { infants: an open-label randomised, } \\
\text { controlled trial }\end{array}$ & 220 & $\begin{array}{l}\text { Surfactant } \\
\text { without } \\
\text { ventilation }\end{array}$ & Standard care & Multiple & $\begin{array}{l}26 \text { to } 28^{+6} \\
\text { weeks }\end{array}$ & Preterm & Respiratory \\
\hline $\begin{array}{l}\text { Harris } 2013 \\
\text { [35] }\end{array}$ & $\begin{array}{l}\text { Dextrose gel for neonatal } \\
\text { hypoglycaemia (the Sugar Babies } \\
\text { study): a randomised, double-blind, } \\
\text { placebo-controlled trial }\end{array}$ & 237 & Dextrose gel & Placebo & Single & $\begin{array}{l}35 \text { to } 42 \\
\text { weeks }\end{array}$ & Both & $\begin{array}{l}\text { Other- } \\
\text { metabolic/ } \\
\text { endocrine }\end{array}$ \\
\hline $\begin{array}{l}\text { Hyttel- } \\
\text { Sorenson } \\
2015 \text { [36] }\end{array}$ & $\begin{array}{l}\text { Cerebral near infrared spectroscopy } \\
\text { oximetry in extremely preterm } \\
\text { infants: phase II randomised clinical } \\
\text { trial }\end{array}$ & 166 & $\begin{array}{l}\text { Cerebral near } \\
\text { infrared } \\
\text { spectroscopy } \\
\text { monitoring }\end{array}$ & $\begin{array}{l}\text { Blinded near } \\
\text { infrared } \\
\text { spectroscopy } \\
\text { monitoring }\end{array}$ & Multiple & $\begin{array}{l}<27^{+6} \\
\text { weeks }\end{array}$ & Preterm & Neurological \\
\hline $\begin{array}{l}\text { Kelleher } \\
2013[37]\end{array}$ & $\begin{array}{l}\text { Oronasopharyngeal suction versus } \\
\text { wiping of the mouth and nose at } \\
\text { birth: a randomised equivalency trial }\end{array}$ & 488 & $\begin{array}{l}\text { Gentle wiping of } \\
\text { the face, mouth } \\
\text { and nose with a } \\
\text { towel }\end{array}$ & $\begin{array}{l}\text { Suction with a } \\
\text { bulb syringe of } \\
\text { the mouth and } \\
\text { nostrils }\end{array}$ & Single & $\geq 35$ weeks & Both & Respiratory \\
\hline $\begin{array}{l}\text { Kimberlin } \\
2011[38]\end{array}$ & $\begin{array}{l}\text { Orally administered acyclovir } \\
\text { suppression and neurodevelopment } \\
\text { after neonatal herpes }\end{array}$ & 74 & Oral acyclovir & Placebo & Multiple & $>800 \mathrm{~g}$ & Both & Infection \\
\hline $\begin{array}{l}\text { Kimberlin } \\
2015[39]\end{array}$ & $\begin{array}{l}\text { Valganciclovir for symptomatic } \\
\text { congenital cytomegalovirus disease }\end{array}$ & 96 & $\begin{array}{l}\text { Valganciclovir } \\
\text { therapy }\end{array}$ & Placebo & Multiple & $\geq 32$ weeks & Both & Infection \\
\hline $\begin{array}{l}\text { Kirpalani } \\
2013[40]\end{array}$ & $\begin{array}{l}\text { A trial comparing non-invasive ventilation } \\
\text { strategies in preterm infants }\end{array}$ & 1007 & $\begin{array}{l}\text { Nasal intermittent } \\
\text { positive-pressure } \\
\text { ventilation }\end{array}$ & $\begin{array}{l}\text { Nasal continuous } \\
\text { positive airway } \\
\text { pressure }\end{array}$ & Multiple & $\begin{array}{l}<30 \text { weeks } \\
\text { and }<1000 \\
g\end{array}$ & Preterm & Respiratory \\
\hline $\begin{array}{l}\text { Leuchter } \\
2014[41]\end{array}$ & $\begin{array}{l}\text { Association between early } \\
\text { administration of high-dose } \\
\text { erythropoietin in preterm infants } \\
\text { and brain magnetic resonance imaging } \\
\text { (MRI) abnormality at term-equivalent } \\
\text { age }\end{array}$ & 165 & $\begin{array}{l}\text { Recombinant } \\
\text { human } \\
\text { erythropoietin }\end{array}$ & Placebo & Multiple & $\begin{array}{l}26 \text { weeks } \\
\text { to } 31^{+6} \\
\text { weeks } \\
\text { +days }\end{array}$ & Preterm & Neurological \\
\hline $\begin{array}{l}\text { Makrides } \\
2009 \text { [42] }\end{array}$ & $\begin{array}{l}\text { Neurodevelopmental outcomes of } \\
\text { preterm infants fed high-dose } \\
\text { docosahexaenoic acid }\end{array}$ & 657 & $\begin{array}{l}\text { High } \\
\text { docosahexaenoic } \\
\text { acid diet }\end{array}$ & $\begin{array}{l}\text { Standard } \\
\text { docosahexaenoic } \\
\text { acid diet }\end{array}$ & Multiple & $<33$ weeks & Preterm & $\begin{array}{l}\text { Other- } \\
\text { nutrition }\end{array}$ \\
\hline $\begin{array}{l}\text { Manley } 2013 \\
{[43]}\end{array}$ & $\begin{array}{l}\text { High-flow nasal cannulae in very } \\
\text { preterm infants after extubation }\end{array}$ & 303 & $\begin{array}{l}\text { High-flow nasal } \\
\text { cannulae }\end{array}$ & $\begin{array}{l}\text { Nasal continuous } \\
\text { positive airway } \\
\text { pressure }\end{array}$ & Multiple & $<32$ weeks & Preterm & Respiratory \\
\hline $\begin{array}{l}\text { Manzoni } \\
2007[44]\end{array}$ & $\begin{array}{l}\text { A multicentre, randomized trial of } \\
\text { prophylactic fluconazole in preterm } \\
\text { neonates }\end{array}$ & 322 & Fluconazole & Placebo & Multiple & $<1500 \mathrm{~g}$ & Preterm & Infection \\
\hline $\begin{array}{l}\text { Manzoni } \\
2009[45]\end{array}$ & $\begin{array}{l}\text { Bovine lactoferrin supplementation } \\
\text { for prevention of late-onset sepsis in } \\
\text { very-low-birth-weight neonates }\end{array}$ & 472 & Lactoferrin & $\begin{array}{l}\text { Lactoferrin + } \\
\text { Lactobacillus } \\
\text { rhamnosus GG } \\
\text { Placebo }\end{array}$ & Multiple & $<1500 \mathrm{~g}$ & Preterm & Infection \\
\hline $\begin{array}{l}\text { Mercier } 2010 \\
{[46]}\end{array}$ & $\begin{array}{l}\text { Inhaled nitric oxide for prevention of } \\
\text { bronchopulmonary dysplasia in } \\
\text { premature babies (EUNO): a } \\
\text { randomised controlled trial }\end{array}$ & 800 & $\begin{array}{l}\text { Inhaled nitric } \\
\text { oxide }\end{array}$ & Placebo & Multiple & $\begin{array}{l}24^{+0} \text { to } \\
28^{+6} \\
\text { weeks }^{+ \text {days }}\end{array}$ & Preterm & Respiratory \\
\hline $\begin{array}{l}\text { Morley } 2008 \\
{[47]}\end{array}$ & $\begin{array}{l}\text { Nasal CPAP or intubation at birth for } \\
\text { very preterm infants }\end{array}$ & 610 & CPAP & $\begin{array}{l}\text { Intubation and } \\
\text { ventilation at } 5 \text { min }\end{array}$ & Multiple & $\begin{array}{l}25^{+0} \text { to } \\
28^{+6} \\
\text { weeks }^{+ \text {days }}\end{array}$ & Preterm & Respiratory \\
\hline $\begin{array}{l}\text { Morris } 2008 \\
{[48]}\end{array}$ & $\begin{array}{l}\text { Aggressive versus conservative } \\
\text { phototherapy for infants with } \\
\text { extremely low birth weight }\end{array}$ & 1974 & $\begin{array}{l}\text { Aggressive } \\
\text { phototherapy }\end{array}$ & $\begin{array}{l}\text { Conservative } \\
\text { phototherapy }\end{array}$ & Multiple & $501-1000 \mathrm{~g}$ & Preterm & Other- hepatic \\
\hline
\end{tabular}


Table 1 The identified studies and their characteristics (Continued)

\begin{tabular}{|c|c|c|c|c|c|c|c|c|}
\hline $\begin{array}{l}\text { Author and } \\
\text { year }\end{array}$ & Title & $N^{a}$ & Intervention arm & Comparator arm & $\begin{array}{l}\text { Single/ } \\
\text { multiple } \\
\text { centre } \\
\text { trial }\end{array}$ & $\begin{array}{l}\text { Age/ } \\
\text { weight } \\
\text { Inclusion } \\
\text { criteria of } \\
\text { participants }\end{array}$ & $\begin{array}{l}\text { Infant } \\
\text { age } \\
\text { group }\end{array}$ & Disease area \\
\hline $\begin{array}{l}\text { Morris } 2013 \\
\text { [49] }\end{array}$ & $\begin{array}{l}\text { Percutaneous vesicoamniotic } \\
\text { shunting versus conservative } \\
\text { management for fetal lower urinary } \\
\text { tract obstruction (PLUTO): a } \\
\text { randomised trial }\end{array}$ & 31 & $\begin{array}{l}\text { Percutaneous } \\
\text { vesicoamniotic } \\
\text { shunting }\end{array}$ & $\begin{array}{l}\text { Conservative } \\
\text { management }\end{array}$ & Multiple & $\begin{array}{l}\text { No age or } \\
\text { weight } \\
\text { criteria }\end{array}$ & Both & Genitourinary \\
\hline $\begin{array}{l}\text { Moss } 2006 \\
{[50]}\end{array}$ & $\begin{array}{l}\text { Laparotomy versus peritoneal } \\
\text { drainage for necrotising enterocolitis } \\
\text { (NEC) and perforation }\end{array}$ & 117 & $\begin{array}{l}\text { Primary peritoneal } \\
\text { drainage }\end{array}$ & $\begin{array}{l}\text { Laparotomy with } \\
\text { bowel resection }\end{array}$ & Multiple & $\begin{array}{l}<34 \text { weeks, } \\
<1500 \mathrm{~g}\end{array}$ & Preterm & Gastrointestinal \\
\hline $\begin{array}{l}\text { Natalucci } \\
2016[51]\end{array}$ & $\begin{array}{l}\text { Effect of early prophylactic high-dose } \\
\text { recombinant human erythropoietin } \\
\text { in very preterm infants on } \\
\text { neurodevelopmental outcome at } 2 \\
\text { years }\end{array}$ & 365 & $\begin{array}{l}\text { Prophylactic early } \\
\text { high-dose recom- } \\
\text { binant human } \\
\text { erythropoietin } \\
\text { (rhEPO) }\end{array}$ & Placebo & Multiple & $\begin{array}{l}26^{+0} \text { to } \\
31^{+6} \\
\text { weeks }\end{array}$ & Preterm & Neurological \\
\hline $\begin{array}{l}\text { Schmidt } \\
2012[52]\end{array}$ & $\begin{array}{l}\text { Survival without disability to age } 5 \\
\text { years after neonatal caffeine therapy } \\
\text { for apnea of prematurity }\end{array}$ & 1640 & Caffeine therapy & Placebo & Multiple & 500-1250 g & Preterm & Respiratory \\
\hline $\begin{array}{l}\text { Schmidt } \\
2013[53]\end{array}$ & $\begin{array}{l}\text { Effects of targeting higher versus } \\
\text { lower arterial oxygen saturations on } \\
\text { death or disability in extremely } \\
\text { preterm infants }\end{array}$ & 1201 & $\begin{array}{l}\text { Oxygen saturation } \\
85-89 \%\end{array}$ & $\begin{array}{l}\text { Oxygen } \\
\text { saturation } 91- \\
95 \%\end{array}$ & Multiple & $\begin{array}{l}23^{+0} \text { to } \\
27^{+6} \\
\text { weeks }\end{array}$ & Preterm & Respiratory \\
\hline $\begin{array}{l}\text { Shankaran } \\
2012[54]\end{array}$ & $\begin{array}{l}\text { Childhood outcomes after } \\
\text { hypothermia for neonatal } \\
\text { encephalopathy }\end{array}$ & 190 & Hypothermia & Usual care & Multiple & $\geq 36$ weeks & Both & Neurological \\
\hline $\begin{array}{l}\text { Shankaran } \\
2014[55]\end{array}$ & $\begin{array}{l}\text { Effect of depth and duration of } \\
\text { cooling on deaths in the neonatal } \\
\text { intensive care unit (NICU) among } \\
\text { neonates with hypoxic ischemic } \\
\text { encephalopathy, a randomised } \\
\text { clinical trial }\end{array}$ & 364 & $\begin{array}{l}32^{\circ} \mathrm{C} \text { for } 72 \mathrm{~h} \\
33.5^{\circ} \mathrm{C} \text { for } 120 \mathrm{~h} \\
32^{\circ} \mathrm{C} \text { for } 120 \mathrm{~h}\end{array}$ & $33.5^{\circ} \mathrm{C}$ for $72 \mathrm{~h}$ & Multiple & $\geq 36$ weeks & Both & Neurological \\
\hline $\begin{array}{l}\text { Slater } 2010 \\
{[56]}\end{array}$ & $\begin{array}{l}\text { Orally administered sucrose as an } \\
\text { analgesic drug for procedural pain in } \\
\text { newborn infants: a randomised } \\
\text { controlled trial }\end{array}$ & 44 & Sucrose solution & Sterile water & Single & $\begin{array}{l}37-43 \\
\text { weeks }\end{array}$ & Term & Other- pain \\
\hline $\begin{array}{l}\text { Stenson } \\
2013[57]\end{array}$ & $\begin{array}{l}\text { Oxygen saturation and outcomes in } \\
\text { preterm infants }\end{array}$ & 2448 & $\begin{array}{l}\text { Oxygen saturation } \\
\text { of } 85-89 \%\end{array}$ & $\begin{array}{l}\text { Oxygen } \\
\text { saturation of } 91- \\
95 \%\end{array}$ & Multiple & $<28$ weeks & Preterm & Respiratory \\
\hline $\begin{array}{l}\text { Taddio } 2006 \\
\text { [58] }\end{array}$ & $\begin{array}{l}\text { Intravenously administered morphine } \\
\text { and topically administered tetracaine } \\
\text { for treatment of pain in preterm } \\
\text { neonates undergoing central-line } \\
\text { placement }\end{array}$ & 132 & $\begin{array}{l}\text { Tetracaine or } \\
\text { morphine or both }\end{array}$ & $\begin{array}{l}\text { Neither tetracaine } \\
\text { nor morphine }\end{array}$ & Multiple & $\begin{array}{l}\text { No age or } \\
\text { weight } \\
\text { criteria }\end{array}$ & Both & Other- pain \\
\hline $\begin{array}{l}\text { Tarnow- } \\
\text { Mordi } 2016 \\
\text { [59] }\end{array}$ & $\begin{array}{l}\text { Outcomes of two trials of oxygen- } \\
\text { saturation targets in preterm infants }\end{array}$ & 1858 & $\begin{array}{l}\text { Lower oxygen- } \\
\text { saturation range }\end{array}$ & $\begin{array}{l}\text { Higher oxygen- } \\
\text { saturation range }\end{array}$ & Multiple & $<28$ weeks & Preterm & Respiratory \\
\hline $\begin{array}{l}\text { Vaucher } \\
2012[60]\end{array}$ & $\begin{array}{l}\text { Neurodevelopmental outcomes in } \\
\text { the early CPAP and pulse oximetry } \\
\text { trial }\end{array}$ & 990 & $\begin{array}{l}\text { Early CPAP with a } \\
\text { limited ventilation } \\
\text { strategy }\end{array}$ & $\begin{array}{l}\text { Early surfactant } \\
\text { administration } \\
(2 \times 2 \text { factorial) } \\
\text { Also to: } 85-89 \% \\
\text { oxygen saturation } \\
\text { or } 91-95 \% \\
\text { oxygen saturation }\end{array}$ & Multiple & $\begin{array}{l}24^{+0} \text { to } \\
27^{+6} \\
\text { weeks }^{+ \text {days }}\end{array}$ & Preterm & Respiratory \\
\hline $\begin{array}{l}\text { Zivanovic } \\
2014[61]\end{array}$ & $\begin{array}{l}\text { Late outcomes of a randomized trial } \\
\text { of high-frequency oscillation in } \\
\text { neonates }\end{array}$ & 319 & $\begin{array}{l}\text { High-frequency } \\
\text { oscillatory } \\
\text { ventilation }\end{array}$ & $\begin{array}{l}\text { Conventional } \\
\text { ventilation }\end{array}$ & Multiple & $<29$ weeks & Preterm & Respiratory \\
\hline
\end{tabular}

${ }^{\mathrm{a}}$ Number of infants presenting baseline characteristics 
Table 2 Data items reported in more than 20\% of studies and stratified by the age of the study participants

\begin{tabular}{|c|c|c|c|c|c|c|c|c|}
\hline \multirow{3}{*}{ Baseline Characteristics } & \multicolumn{6}{|c|}{ Infant age } & \multirow{2}{*}{\multicolumn{2}{|c|}{$\begin{array}{l}\text { All studies } \\
(n=44)\end{array}$}} \\
\hline & \multicolumn{2}{|c|}{$\begin{array}{l}\text { Preterm studies } \\
(n=29)\end{array}$} & \multicolumn{2}{|c|}{$\begin{array}{l}\text { Term studies } \\
(n=6)\end{array}$} & \multicolumn{2}{|c|}{$\begin{array}{l}\text { Mixed-ages studies } \\
(n=9)\end{array}$} & & \\
\hline & & & & & & & & \\
\hline Gestational age & 29 & $(100 \%)$ & 4 & $(67 \%)$ & 9 & $(100 \%)$ & 42 & $(96 \%)$ \\
\hline Sex & 29 & $(100 \%)$ & 6 & $(100 \%)$ & 6 & $(67 \%)$ & 41 & $(93 \%)$ \\
\hline Birth weight & 29 & $(100 \%)$ & 5 & $(83 \%)$ & 6 & $(67 \%)$ & 40 & (91\%) \\
\hline Antenatal steroids & 25 & $(86 \%)$ & 1 & $(17 \%)$ & 1 & $(11 \%)$ & 27 & $(61 \%)$ \\
\hline Multiple births & 21 & $(72 \%)$ & 1 & $(17 \%)$ & 2 & $(22 \%)$ & 24 & $(55 \%)$ \\
\hline Respiratory support & 17 & $(59 \%)$ & 3 & $(50 \%)$ & 3 & $(33 \%)$ & 23 & $(52 \%)$ \\
\hline Mode of delivery & 14 & $(48 \%)$ & 2 & $(33 \%)$ & 5 & $(56 \%)$ & 21 & $(48 \%)$ \\
\hline Infection & 15 & $(52 \%)$ & 3 & $(50 \%)$ & 3 & (33\%) & 21 & $(48 \%)$ \\
\hline Drug treatment & 15 & $(52 \%)$ & 0 & (0\%) & 5 & $(56 \%)$ & 20 & $(45 \%)$ \\
\hline Maternal ethnicity & 15 & $(52 \%)$ & 1 & $(17 \%)$ & 3 & (33\%) & 19 & $(43 \%)$ \\
\hline Apgar score 5 min & 14 & $(48 \%)$ & 0 & $(0 \%)$ & 5 & $(56 \%)$ & 19 & $(43 \%)$ \\
\hline Age & 11 & $(38 \%)$ & 6 & $(100 \%)$ & 2 & $(22 \%)$ & 19 & $(43 \%)$ \\
\hline Inborn & 13 & $(45 \%)$ & 0 & $(0 \%)$ & 2 & $(22 \%)$ & 15 & $(34 \%)$ \\
\hline Maternal age & 6 & $(21 \%)$ & 1 & $(17 \%)$ & 6 & $(67 \%)$ & 13 & $(30 \%)$ \\
\hline \multicolumn{9}{|l|}{ Stratification items } \\
\hline Neonatal unit identifier & 22 & $(76 \%)$ & 1 & $(17 \%)$ & 2 & $(22 \%)$ & 25 & $(57 \%)$ \\
\hline Gestational age & 14 & $(48 \%)$ & 1 & $(17 \%)$ & 3 & $(33 \%)$ & 17 & $(39 \%)$ \\
\hline \multicolumn{9}{|c|}{ Primary outcome adjusting items } \\
\hline Gestational age & 17 & $(59 \%)$ & 1 & $(8 \%)$ & 1 & $(11 \%)$ & 19 & $(43 \%)$ \\
\hline Neonatal unit identifier & 10 & $(34 \%)$ & 1 & $(8 \%)$ & 2 & $(22 \%)$ & 13 & $(28 \%)$ \\
\hline Birth weight & 9 & (31\%) & 0 & (0\%) & 1 & (11\%) & 10 & (22\%) \\
\hline
\end{tabular}

during randomisation. Two (13\%) of these stratification items were reported by more than $20 \%$ of trials and 9 (56\%) were reported by one study only (Additional tables). Twenty-four items were reported by 33 trials to adjust the primary outcome. Of these, $3(13 \%)$ were reported by more than $20 \%$ of all trials and $12(50 \%)$ were reported by one study only (Additional file 2 Tables S1, S2, S3, S4). Eight (50\%) stratification and 9 (38\%) adjustment items were in the top 14 background data items. A full list of all common items can be found in the Additional file 2 Tables S1, S2, S3, S4.

\section{Data completeness}

In 2015 , 96,699 infants were admitted to 180 neonatal units in England, Wales and Scotland. Admitted infants received 472,187 days of neonatal care during the first 7 days following birth (data not shown).

The completeness of common data items in the NNRD are summarised by age groups in Table 3. Data completeness in the NNRD is $99.9 \%$ for gestational age at birth, $99.9 \%$ for sex, $100 \%$ for birth weight, $99.7 \%$ for multiple birth and $100 \%$ for respiratory support on day 1 (Table 3). The majority of data items were more than
90\% complete, exceptions include maternal ethnicity (70.2\%), mode of delivery (81.4\%) and Apgar score at 5 min (79.1\%). Completeness was higher for all data items for preterm (mean completeness 94.4\%) compared to term babies (mean completeness 89.2\%) (Table 3).

\section{Discussion}

We have identified a common set of non-outcome data items reported in high-impact neonatal trials. We find that 12 of these 14 data items can be obtained from the NNRD with high completeness for most items (Table 3). The common data items identified here have previously been validated against independently collected trial data [17] where they were shown to be highly accurate and complete in the NNRD. This supports the assertion that non-outcome data held in the NNRD can be used to support large, efficient neonatal trials. We recognise that the trials included in the systematic review also reported a wide range of additional non-outcome data items that were not included in the common set identified here. In planning future pragmatic neonatal trials, the completeness and accuracy of additional data items critical to the integrity of a planned trial can be evaluated using 
Table 3 Data completeness in the National Neonatal Research Database (NNRD) for the data items reported in 20\% of studies or more

\begin{tabular}{|c|c|c|c|c|}
\hline & \multicolumn{3}{|l|}{ Age } & \multirow[b]{2}{*}{$\begin{array}{l}\text { All } \\
(n=96,699) \\
(\%)\end{array}$} \\
\hline & $\begin{array}{l}\text { Preterm } \\
(n=37,424) \\
(\%)\end{array}$ & $\begin{array}{l}\text { Term } \\
(n=59,130) \\
(\%)\end{array}$ & $\begin{array}{l}\text { Unknown } \\
(n=145) \\
(\%)\end{array}$ & \\
\hline Gestational age & 100.0 & 100.0 & 0 & 99.9 \\
\hline Sex & 99.9 & 99.9 & 99.3 & 99.9 \\
\hline Birth weight & 100.0 & 100.0 & 91.7 & 100.0 \\
\hline Antenatal steroids & 94.5 & 89.7 & 4.8 & 91.4 \\
\hline Maternal ethnicity & 75.6 & 66.9 & 1.4 & 70.2 \\
\hline Multiple births & 100.0 & 99.8 & 11.7 & 99.7 \\
\hline Mode of delivery & 90.7 & 75.7 & 2.8 & 81.4 \\
\hline Apgar score at $5 \mathrm{~min}$ & 87.6 & 73.9 & 0.7 & 79.1 \\
\hline Maternal age & 96.6 & 89.2 & 3.4 & 92.0 \\
\hline Inborn ${ }^{a}$ & 98.8 & 96.6 & 6.2 & 97.3 \\
\hline Drug treatment in the first 1 day ${ }^{b c}$ & & & & 91.9 \\
\hline Respiratory support in the first 1 day ${ }^{c}$ & & & & 100.0 \\
\hline
\end{tabular}

${ }^{a}$ Corresponding NNRD data item: place of birth

${ }^{\mathrm{b}}$ Corresponding NNRD data item: any medication recorded on day 1 of admission

${ }^{\mathrm{C}}$ For babies less than 28 weeks gestational age $(n=1967)$

approaches similar to those applied here. However, the finding that reported data items were variable even between similar trials (Additional file 2: Table S2) suggests that some reported data items may not have been critical to trial integrity, and that harmonisation of nonoutcome data items may improve the consistency and efficiency of future neonatal trials. The common nonoutcome data items we identify here, and their completeness and accuracy [17] in the NNRD, can be used to assess the suitability and feasibility of using the NNRD and other similar routinely recorded data sources for neonatal trials.

Data completeness of the NNRD has previously been calculated by Battersby et al. [17] in relation to a single clinical trial between 2008 and 2015. In this study percentage completeness was very similar to that found in the present study where common data items examined multiple births, gestational age, sex and birth weight, indicating that data completeness within the NNRD for these items is consistent over time. The present study builds upon this work by examining completeness for a wider range of empirically identified non-outcome data items; therefore, extending the relevance of these results to a wider range of potential clinical trials. For large neonatal trials in the United Kingdom, we demonstrate that the core non-outcome data items identified here are held in the NNRD to a high degree of completeness. For some core non-outcome data items, such as gestational age at birth, we show that the likelihood of missing data in clinical trials utilising the NNRD is small. These results can be used to develop and apply approaches to improve the recording of critical data items with lower completeness in a targeted way; for example, mode of delivery.

Common datasets in other clinical and research areas have been identified using a variety of methods. Doods et al. [62] identified common data groups and elements for feasibility analysis in cardiovascular medicine, diabetes, inflammatory, oncology and neurology through the use of an expert panel, but did not review the literature or include expertise from outside the field. This study identified a wide range of laboratory tests for feasibility studies. Diagnostic test data were not identified in our systematic review of large neonatal trials as commonly reported non-outcome data items, indicating that such data items are not as relevant to the pragmatic neonatal trials that are the focus of this work. Sheehan at al [63]. outline previously developed common data element sets, and some of the challenges inherent in adopting and using such sets. Chari et al. [64] conducted a systematic review of included trials and observational studies to identify common data elements in chronic subdural haematoma studies and, in keeping with our results, identified a core set of commonly reported nonoutcome items. The approach that we used was a more limited systematic review of trials published in highimpact journals. This approach was chosen a-priori to focus on data items reported in trials that influence neonatal practice. This was a pragmatic decision and there are limitations to this approach: by limiting our review to general medical journals we may have missed influential trials published in specialty journals, and have not 
sampled the range of outcomes reported in smaller trials. Furthermore, no approach to date has sought parent or patient views on the importance of different nonoutcome data items; this may be important given the different priorities identified by these groups compared to health professionals and researchers [65]. The examples cited here demonstrate the interest in, and potential value of, common sets of non-outcome data items, across different specialties. The development of an established methodological approach, analogous to that developed by the COMET initiative [12] would increase the consistency, robustness and comparability of such endeavours in future.

Our study has focussed on defining the data items usually recorded at baseline or used as explanatory data items in clinical trials. To our best knowledge there have been no previous attempts to identify core non-outcome trial data items such as these. We included the most common data items used in randomisation, which are often selected to conduct pre-specified subgroup analyses, and to adjust for the primary outcome. These items are often overlooked when exploring the impact of data quality in trials, despite the importance of completeness of these items for preserving statistical power and avoiding misinterpretation of results. We did not focus on outcome data items because the methodology to identify these data is well developed and such work is underway in neonatal medicine [16]. A limitation of our study is that data may have been selectively reported thus introducing bias; however, this is lessened as the included journal review protocols are designed to ensure that those items listed in the protocol are presented in the main trial outcomes publication. A further limitation of our study was that some items identified were dichotomous; for example, presence or absence of infection prior to trial enrolment and it was not possible to calculate completeness for such items as absence of the condition is not always actively recorded. Age was found to be a common data item; however, it is calculated using gestational age which is highly complete in the NNRD and, therefore, completeness for age was not calculated. An additional limitation stems from the fact that some data items collected in clinical trials did not directly align with data items in the NNRD; therefore, there may be a loss of information from aggregating several data items into a common data item held by the NNRD to assess data quality. Furthermore, included trials used different approaches to ascertain commonly reported data items; for example, the most commonly reported data item - gestational age - may be derived from maternal reported data, ultrasound measurement or clinical evaluation. Data held within the NNRD are extracted from routine clinical information used to inform clinical care, these clinically relevant data may be more appropriate for pragmatic trials than more granular data items reported in trials. Differences between trials and routinely recorded data sources in how data items are ascertained and synthesised have the potential to introduce biases into clinical trials seeking to use such routinely recorded data. Where such differences are randomly distributed between trial arms, the impact may be limited to lower precision, rather than systematic bias in favour of one trial arm. Further exploration is needed to understand how to accurately assess and synthesise similar data items and to quantify the direction and magnitude of potential biases.

It is important to note that some NNRD data items had between 10 and 30\% missing data. The implications of such degrees of missingness depend on the role of the data item in the trial, but are likely to lead to a loss of precision [66]. Baseline variables have a role in prespecified statistical analyses of outcomes in order that treatment effects can be estimated more precisely. Where the baseline is missing, there are methods which do allow incomplete baseline variables to be included without removing the patients with missing baselines, and to achieve some increase in precision. This is relevant to individually randomised trials, whereas an incomplete baseline may have a greater impact in trials randomising centre clusters when baseline completeness varies by centre. Baseline variables are also used to describe the trial population; for example, to allow readers to judge generalisability, and a high level of baseline completeness may be important for this purpose. Finally, baseline variables are important for subgroup analyses and missing data may limit such analyses. The results presented here will allow the impact that different degrees of missingness have in neonatal trials to be further explored and modelled to better understand which trials are most suitable to use routinely recorded data. The more widespread use of routinely collected data for clinical trials also has the potential to improve the recording of such data [67]. Another limitation is that we did not evaluate the accuracy of common non-outcome data items in the NNRD in this study, although this has recently been undertaken [17]. Completeness and accuracy are key factors in determining the suitability of using routinely recorded clinical data for clinical trials and should be evaluated for all data items deemed critical to any trial seeking to use such data.

The clinical and economic efficiency of using routinely recorded common data items has been demonstrated by trials that have used common registries such as SWEDEHEART $[68,69]$. Common data items, as identified here and in core outcome sets [70], can be used to ensure that existing primary data capture systems such as EPR systems and registries capture appropriate data for trials, and in planning such trials. High accuracy and 
completeness of data are critical for trials; it may, however, not be feasible to evaluate such metrics for all data items within a database or registry - common data items and core outcome sets can be used to target quality assessment of data items most critical to a range of clinical trials. Ongoing data-enabled pilot trials that use routinely recorded data held in the NNRD (15) should provide prospective data regarding the feasibility of such an approach in the neonatal field.

\section{Conclusion}

Neonatal trials in high-impact journals report a common set of non-outcome data items in their primary publications. In the UK, our study indicates that these core non-outcome data can be obtained from the NNRD; the feasibility and efficiency using routinely recorded EPR data such as that held in the NNRD for neonatal clinical trials, rather than collecting these items anew, should be examined. We suggest that when planning primary data collection systems such as EPR systems, registries or clinical databases, consideration is given to fostering a culture of completeness and ensuring that important items are accurately and completely captured.

\section{Supplementary information}

Supplementary information accompanies this paper at https://doi.org/10 1186/s13063-019-3849-7.

\section{Additional file 1. NeoCODE PubMed search strategy.}

Additional file 2: Table S1. All baseline data items reported by the studies stratified by whether the study recruited preterm or term infants. Data items refer to infant chanracteristics unless otherwise stated. Table S2. The most common baseline data items by each identified study. Black indicates that the study presented the data item at baseline. Table S3. All data items used as stratifying items during randomisation reported by the studies and by the age of infants included in the studies. Table S4. All data items used as confounders to adjust the primary outcome reported by the studies and by the age of infants included in the studies

\section{Abbreviations}

caDSR: Cancer Data Standards Registry; EPR: Electronic Patient Records; NDAU: Neonatal Data Analysis Unit; NHS: National Health Service; NNRD: National Neonatal Research Database; PROMIS: Patient Reported Outcomes Measurement Information System

\section{Acknowledgements}

Daniel Gray (data analyst and reporting specialist, Clevermed Ltd.) provided data support.

\section{Authors' contributions}

CG and SJ conceived this project. CG and SJ undertook data extraction. SJ analysed data and drafted the first draft of this manuscript. All authors contributed to and revised the manuscript. All authors read and approved the final manuscript.

\section{Funding}

This study is part of a PhD funded by a tuition fees grant from the Westminster Medical School Research Trust awarded to Sena Jawad. Sena Jawad and Chris Gale are supported by a Medical Research Council Clinician Scientist Fellowship awarded to Chris Gale.

\section{Availability of data and materials}

The datasets analysed during the current study are available in the National Neonatal Research Database; https://www.imperial.ac.uk/neonatal-dataanalysis-unit/neonatal-data/utilising-the-nnrd/

\section{Ethics approval and consent to participate}

The National Neonatal Research Database has Research Ethics Approval (London Queen Square Research Ethics Committee Reference number 16/ LO/1930).

\section{Consent for publication}

Not applicable

\section{Competing interests}

NM is Director of the Neonatal Data Analysis Unit that created and manages the NNRD. CG and NM are voluntary, unremunerated members of the Neonatal Data Analysis Unit (NDAU) Steering Board which oversees the NNRD.

\section{Author details}

${ }^{1}$ Neonatal Medicine, School of Public Health, Faculty of Medicine, Imperial College London, Chelsea and Westminster Hospital Campus, London SW10 $9 \mathrm{NH}$, UK. ${ }^{2}$ Imperial Clinical Trials Unit, School of Public Health, Imperial College London, London W12 7RH, UK.

Received: 23 April 2019 Accepted: 25 October 2019

Published online: 16 December 2019

\section{References}

1. Sinclair JC, Haughton DE, Bracken MB, Horbar JD, Soll RF. Cochrane neonatal systematic reviews: a survey of the evidence for neonatal therapies. Clin Perinatol. 2003;30(2):285-304.

2. Willhelm C, Girisch W, Gottschling S, Graber S, Wahl H, Meyer S. Systematic Cochrane reviews in neonatology: a critical appraisal. Pediatr Neonatol. 2013;54(4):261-6.

3. Collier R. Rapidly rising clinical trial costs worry researchers. CMAJ. 2009; 180(3):277-8.

4. Speich B, von Niederhausern B, Schur N, Hemkens LG, Furst T, Bhatnagar N, et al. Systematic review on costs and resource use of randomized clinical trials shows a lack of transparent and comprehensive data. J Clin Epidemiol. 2018;96:1-11.

5. NIHR. NIHR Journals Library 2019. Available from: https://www. journalslibrary.nihr.ac.uk.

6. Le Jeannic A, Quelen C, Alberti C, Durand-Zaleski I, CompaRec I. Comparison of two data collection processes in clinical studies: electronic and paper case report forms. BMC Med Res Methodol. 2014;14:7.

7. Lauer MS, D'Agostino RB Sr. The randomized registry trial一the next disruptive technology in clinical research? N Engl J Med. 2013;369(17): 1579-81.

8. Grossmann C, Sanders J, English RA. Large simple trials and knowledge generation in a learning healthcare system. In: Institute of Medicine. Washington D.C.: The National Academies Press; 2013.

9. Eapen ZJ, Lauer MS, Temple RJ. The imperative of overcoming barriers to the conduct of large, simple trials. JAMA. 2014:311(14):1397-8.

10. Ford I, Norrie J. Pragmatic trials. N Engl J Med. 2016;375(5):454-63.

11. Kopcke F, Trinczek B, Majeed RW, Schreiweis B, Wenk J, Leusch T, et al. Evaluation of data completeness in the electronic health record for the purpose of patient recruitment into clinical trials: a retrospective analysis of element presence. BMC Med Inform Decis Mak. 2013:13:37.

12. Williamson PR, Altman DG, Bagley H, Barnes KL, Blazeby JM, Brookes ST, et al. The COMET Handbook: version 1.0. Trials. 2017;18(Suppl 3):280.

13. Digital N. National Neonatal Data Set NHS Data Dictionary; [Available from: https://www.datadictionary.nhs.uk/data_dictionary/messages/dinical_data_ sets/overviews/national_neonatal_data_set_overviews/national_neonatal_ data_set_introduction.asp?shownav=1?query=\%22national +neonatal\%22 \&rank=100\&shownav=1. Accessed 16 Oct 2019.

14. Gale C, Modi N, Jawad S, Culshaw L, Dorling J, Bowler U, et al. The WHEAT pilot trial-WithHolding Enteral feeds Around packed red cell Transfusion to prevent necrotising enterocolitis in preterm neonates: a multicentre, electronic patient record (EPR), randomised controlled point-of-care pilot trial. BMJ Open. 2019;9(9):e033543. 
15. Reuters T. InCites Journal Citation Reports [Available from: https://jcr.incites. thomsonreuters.com. Accessed 16 Oct 2019

16. Webbe J, Brunton G, Ali S, Duffy JMN, Modi N, Gale C. Developing, implementing and disseminating a core outcome set for neonatal medicine. BMJ Paediatrics Open. 2017;1:e000048.

17. Battersby C, Statnikov Y, Santhakumaran S, Gray D, Modi N, Costeloe K, et al. The United Kingdom National Neonatal Research Database: a validation study. PLoS One. 2018;13(8):e0201815.

18. Azzopardi DV, Strohm B, Edwards AD, Dyet L, Halliday HL, Juszczak E, et al. Moderate hypothermia to treat perinatal asphyxial encephalopathy. $\mathrm{N}$ Engl J Med. 2009;361(14):1349-58.

19. Azzopardi D, Strohm B, Marlow N, Brocklehurst P, Deierl A, Eddama O, et al. Effects of hypothermia for perinatal asphyxia on childhood outcomes. $N$ Engl J Med. 2014;371(2):140-9.

20. Ballard RA, Truog WE, Cnaan A, Martin RJ, Ballard PL, Merrill JD, et al. Inhaled nitric oxide in preterm infants undergoing mechanical ventilation. $N$ Engl J Med. 2006;355(4):343-53.

21. Bassler D, Plavka R, Shinwell ES, Hallman M, Jarreau PH, Carnielli V, et al. Early inhaled budesonide for the prevention of bronchopulmonary dysplasia. N Engl J Med. 2015;373(16):1497-506.

22. Baud O, Maury L, Lebail F, Ramful D, El Moussawi F, Nicaise C, et al. Effect of early low-dose hydrocortisone on survival without bronchopulmonary dysplasia in extremely preterm infants (PREMILOC): a double-blind, placebo-controlled, multicentre, randomised trial. Lancet. 2016;387(10030):1827-36

23. Beardsall K, Vanhaesebrouck S, Ogilvy-Stuart AL, Vanhole C, Palmer CR, van Weissenbruch $M$, et al. Early insulin therapy in very-low-birth-weight infants. N Engl J Med. 2008;359(18):1873-84.

24. Benjamin DK Jr, Hudak ML, Duara S, Randolph DA, Bidegain M, Mundakel GT, et al. Effect of fluconazole prophylaxis on candidiasis and mortality in premature infants: a randomized clinical trial. JAMA. 2014;311(17):1742-9.

25. Brocklehurst $P$, Farrell B, King A, Juszczak E, Darlow B, Haque K, et al. Treatment of neonatal sepsis with intravenous immune globulin. N Engl J Med. 2011;365(13):1201-11.

26. Carlo WA, Finer NN, Walsh MC, Rich W, Gantz MG, Laptook AR, et al. Target ranges of oxygen saturation in extremely preterm infants. N Engl J Med. 2010;362(21):1959-69.

27. Carr R, Brocklehurst P, Dore CJ, Modi N. Granulocyte-macrophage colony stimulating factor administered as prophylaxis for reduction of sepsis in extremely preterm, small for gestational age neonates (the PROGRAMS trial): a single-blind, multicentre, randomised controlled trial. Lancet. 2009; 373(9659):226-33.

28. Ceelie I, de Wildt SN, van Dijk M, van den Berg MM, van den Bosch GE, Duivenvoorden $\mathrm{HJ}$, et al. Effect of intravenous paracetamol on postoperative morphine requirements in neonates and infants undergoing major noncardiac surgery: a randomized controlled trial. JAMA. 2013;309(2): $149-54$.

29. Costeloe K, Hardy P, Juszczak E, Wilks M, Millar MR, Study PPI. Bifidobacterium breve BBG-001 in very preterm infants: a randomised controlled phase 3 trial. Lancet. 2016;387(10019):649-60.

30. Davidson AJ, Disma N, de Graaff JC, Withington DE, Dorris L, Bell G, et al Neurodevelopmental outcome at 2 years of age after general anaesthesia and awake-regional anaesthesia in infancy (GAS): an international multicentre, randomised controlled trial. Lancet. 2016;387(10015):239-50.

31. Fergusson DA, Hebert P, Hogan DL, LeBel L, Rouvinez-Bouali N, Smyth JA, et al. Effect of fresh red blood cell transfusions on clinical outcomes in premature, very low-birth-weight infants The ARIPI randomized trial. JAMA. 2012;308(14):1443-51.

32. Finer NN, Carlo WA, Walsh MC, Rich W, Gantz MG, Laptook AR, et al. Early CPAP versus surfactant in extremely preterm infants. N Engl J Med. 2010; 362(21):1970-9.

33. Fivez T, Kerklaan D, Mesotten D, Verbruggen S, Wouters PJ, Vanhorebeek I, et al. Early versus late parenteral nutrition in critically ill children. N Engl J Med. 2016:374(12):1111-22.

34. Gopel W, Kribs A, Ziegler A, Laux R, Hoehn T, Wieg C, et al. Avoidance of mechanical ventilation by surfactant treatment of spontaneously breathing preterm infants (AMV): an open-label, randomised, controlled trial. Lancet. 2011;378(9803):1627-34.

35. Harris DL, Weston PJ, Signal M, Chase JG, Harding JE. Dextrose gel for neonatal hypoglycaemia (the Sugar Babies Study): a randomised, doubleblind, placebo-controlled trial. Lancet. 2013;382(9910):2077-83.
36. Hyttel-Sorensen S, Pellicer A, Alderliesten T, Austin T, van Bel F, Benders M, et al. Cerebral near infrared spectroscopy oximetry in extremely preterm infants: phase II randomised clinical trial. BMJ. 2015;350:g7635.

37. Kelleher J, Bhat R, Salas AA, Addis D, Mills EC, Mallick H, et al. Oronasopharyngeal suction versus wiping of the mouth and nose at birth: a randomised equivalency trial. Lancet. 2013;382(9889):326-30.

38. Kimberlin DW, Whitley RJ, Wan W, Powell DA, Storch G, Ahmed A, et al. Oral acyclovir suppression and neurodevelopment after neonatal herpes. N Engl Jed. 2011;365(14):1284-92

39. Kimberlin DW, Jester PM, Sanchez PJ, Ahmed A, Arav-Boger R, Michaels MG, et al. Valganciclovir for symptomatic congenital cytomegalovirus disease. N Engl J Med. 2015;372(10):933-43.

40. Kirpalani H, Millar D, Lemyre B, Yoder BA, Chiu A, Roberts RS, et al. A trial comparing noninvasive ventilation strategies in preterm infants. N Engl J Med. 2013;369(7):611-20.

41. Leuchter RHV, Gui L, Poncet A, Hagmann C, Lodygensky GA, Martin E, et al. Association between early administration of high-dose erythropoietin in preterm infants and brain MRI abnormality at term-equivalent age. JAMA. 2014;312(8):817-24.

42. Makrides M, Gibson RA, McPhee AJ, Collins CT, Davis PG, Doyle LW, et al. Neurodevelopmental outcomes of preterm infants fed high-dose docosahexaenoic acid a randomized controlled trial. JAMA. 2009;301(2): 175-82.

43. Manley BJ, Owen LS, Doyle LW, Andersen CC, Cartwright DW, Pritchard MA, et al. High-flow nasal cannulae in very preterm infants after extubation. $\mathrm{N}$ Engl J Med. 2013;369(15):1425-33.

44. Manzoni P, Stolfi I, Pugni L, Decembrino L, Magnani C, Vetrano G, et al. A multicenter, randomized trial of prophylactic fluconazole in preterm neonates. N Engl J Med. 2007;356(24):2483-95.

45. Manzoni P, Rinaldi M, Cattani S, Pugni L, Romeo MG, Messner $\mathrm{H}$, et al. Bovine lactoferrin supplementation for prevention of late-onset sepsis in very low-birth-weight neonates: a randomized trial. JAMA. 2009;302(13): 1421-8.

46. Mercier JC, Hummler H, Durrmeyer X, Sanchez-Luna M, Carnielli V, Field D, et al. Inhaled nitric oxide for prevention of bronchopulmonary dysplasia in premature babies (EUNO): a randomised controlled trial. Lancet. 2010; 376(9738):346-54.

47. Morley CJ, Davis PG, Doyle LW, Brion LP, Hascoet JM, Carlin JB, et al. Nasal CPAP or intubation at birth for very preterm infants. N Engl J Med. 2008; 358(7):700-8.

48. Morris BH, Oh W, Tyson JE, Stevenson DK, Phelps DL, O'Shea TM, et al. Aggressive vs. conservative phototherapy for infants with extremely low birth weight. N Engl J Med. 2008;359(18):1885-96.

49. Morris RK, Malin GL, Quinlan-Jones E, Middleton LJ, Hemming K, Burke D, et al. Percutaneous vesicoamniotic shunting versus conservative management for fetal lower urinary tract obstruction (PLUTO): a randomised trial. Lancet. 2013;382(9903):1496-506.

50. Moss RL, Dimmitt RA, Barnhart DC, Sylvester KG, Brown RL, Powell DM, et al. Laparotomy versus peritoneal drainage for necrotizing enterocolitis and perforation. N Engl J Med. 2006;354(21):2225-34.

51. Natalucci G, Latal B, Koller B, Rugger C, Sick B, Held L, et al. Effect of early prophylactic high-dose recombinant human erythropoietin in very preterm infants on neurodevelopmental outcome at 2 years. A randomized clinical trial. JAMA. 2016;315(19):2079-85.

52. Schmidt B, Anderson PJ, Doyle LW, Dewey D, Grunau RE, Asztalos EV, et al. Survival without disability to age 5 years after neonatal caffeine therapy for apnea of prematurity. JAMA. 2012;307(3):275-82.

53. Schmidt B, Whyte RK, Asztalos EV, Moddemann D, Poets C, Rabi Y, et al. Effects of targeting higher vs lower arterial oxygen saturations on death or disability in extremely preterm infants: a randomized clinical trial. JAMA. 2013;309(20):2111-20.

54. Shankaran S, Pappas A, McDonald SA, Vohr BR, Hintz SR, Yolton K, et al. Childhood outcomes after hypothermia for neonatal encephalopathy. N Engl J Med. 2012;366(22):2085-92.

55. Shankaran S, Laptook AR, Pappas A, McDonald SA, Das A, Tyson JE, et al. Effect of depth and duration of cooling on deaths in the NICU among neonates with hypoxic ischemic encephalopathy. A randomized clinical trial. JAMA. 2014;312(24):2629-39.

56. Slater R, Cornelissen L, Fabrizi L, Patten D, Yoxen J, Worley A, et al. Oral sucrose as an analgesic drug for procedural pain in newborn infants: a randomised controlled trial. Lancet. 2010;376(9748):1225-32. 
57. Stenson BJ, Tarnow-Mordi WO, Darlow BA, Simes J, Juszczak E, Askie L, et al. Oxygen saturation and outcomes in preterm infants. N Engl J Med. 2013; 368(22):2094-104.

58. Taddio A, Lee C, Yip A, Parvez B, McNamara PJ, Shah V. Intravenous morphine and topical tetracaine for treatment of pain in preterm neonates undergoing central line placement. JAMA. 2006;295(7):793-800.

59. Tarnow-Mordi W, Stenson B, Kirby A, Juszczak E, Donoghoe M, Deshpande $S$, et al. Outcomes of two trials of oxygen-saturation targets in preterm infants. N Engl J Med. 2016;374(8):749-60.

60. Vaucher YE, Peralta-Carcelen M, Finer NN, Carlo WA, Gantz MG, Walsh MC, et al. Neurodevelopmental outcomes in the early CPAP and pulse oximetry trial. N Engl J Med. 2012;367(26):2495-504.

61. Zivanovic S, Peacock J, Alcazar-Paris M, Lo JW, Lunt A, Marlow N, et al. Late outcomes of a randomized trial of high-frequency oscillation in neonates. $\mathrm{N}$ Engl J Med. 2014;370(12):1121-30.

62. Doods J, Botteri F, Dugas M, Fritz F, Ehr4Cr WP. A European inventory of common electronic health record data elements for clinical trial feasibility. Trials. 2014;15:10

63. Sheehan J, Hirschfeld S, Foster E, Ghitza U, Goetz K, Karpinski J, et al. Improving the value of clinical research through the use of Common Data Elements. Clin Trials. 2016;13(6):671-6.

64. Chari A, Hocking KC, Edlmann E, Turner C, Santarius T, Hutchinson PJ, et al. Core outcomes and common data elements in chronic subdural hematoma: a systematic review of the literature focusing on baseline and peri-operative care data elements. J Neurotrauma. 2016;33(17):1569-75.

65. Webbe J, Brunton G, Ali S, Longford N, Modi N, Gale C, et al. Parent, patient and clinician perceptions of outcomes during and following neonatal care: a systematic review of qualitative research. BMJ Paediatr Open. 2018;2(1):e000343.

66. White IR, Thompson SG. Adjusting for partially missing baseline measurements in randomized trials. Stat Med. 2005;24(7):993-1007.

67. Gale C, Modi N, group Wtd. Neonatal randomised point-of-care trials are feasible and acceptable in the UK: results from two national surveys. Arch Dis Child Fetal Neonatal Ed. 2016;101(1):F86-7.

68. Frobert O, Lagergvist B, Olivecrona GK, Omerovic E, Gudnason T, Maeng M, et al. Thrombus aspiration during ST-segment elevation myocardial infarction. N Engl J Med. 2013:369(17):1587-97.

69. Hofmann R, James SK, Jernberg T, Lindahl B, Erlinge D, Witt N, et al. Oxygen therapy in suspected acute myocardial infarction. N Engl J Med. 2017; 377(13):1240-9.

70. Webbe J, Sinha I, Gale C. Core outcome sets. Arch Dis Child Educ Pract Ed. 2018;103(3):163-6.

\section{Publisher's Note}

Springer Nature remains neutral with regard to jurisdictional claims in published maps and institutional affiliations.

Ready to submit your research? Choose BMC and benefit from:

- fast, convenient online submission

- thorough peer review by experienced researchers in your field

- rapid publication on acceptance

- support for research data, including large and complex data types

- gold Open Access which fosters wider collaboration and increased citations

- maximum visibility for your research: over $100 \mathrm{M}$ website views per year

At $\mathrm{BMC}$, research is always in progress.

Learn more biomedcentral.com/submissions 